(2) Open Access Full Text Article

\title{
Apotemnophilia, body integrity identity disorder or xenomelia? Psychiatric and neurologic etiologies face each other
}

This article was published in the following Dove Press journal:

Neuropsychiatric Disease and Treatment

7 July 2014

Number of times this article has been viewed

\author{
Anna Sedda ${ }^{1,2}$ \\ Gabriella Bottini ${ }^{1,2}$ \\ 'Department of Behavioral and \\ Brain Sciences, University of Pavia, \\ Pavia, ${ }^{2}$ Cognitive Neuropsychology \\ Laboratory, Niguarda Ca' Granda \\ Hospital, Milan, Italy
}

\begin{abstract}
This review summarizes the available studies of a rare condition in which individuals seek the amputation of a healthy limb or desire to be paraplegic. Since 1977, case reports and group studies have been produced, trying to understand the cause of this unusual desire. The main etiological hypotheses are presented, from the psychological/psychiatric to the most recent neurologic explanation. The paradigms adopted and the clinical features are compared across studies and analyzed in detail. Finally, future directions and ethical implications are discussed. A proposal is made to adopt a multidisciplinary approach that comprises state-of-the-art technologies and a variety of theoretical models, including both body representation and psychological and sexual components.
\end{abstract}

Keywords: BIID, limb amputation, somatoparaphrenia, body representation, body ownership

\section{Introduction}

Interacting with the environment to achieve our goals implies mainly motor actions, such as grasping objects or walking to change position. Thanks to these actions, we can drink water when thirsty and leave a room if we see a fire starting. Control over the environment is highly diminished by physical damage to the body: everyday life activities of people with spinal cord injuries or limb amputations, for example, become more challenging. Not surprisingly, most people fear the idea of losing a leg or an arm, or any other violation of the body's external form. ${ }^{1}$

Recent studies show that this preserving behavior is reduced in some individuals who desire the amputation of a healthy limb, instead of actively avoiding body damage. ${ }^{2}$ Importantly, in most individuals, the target limb for amputation is not affected by sensory impairments, such as severe pain or sensory defects (McGeoch et al 2011, ${ }^{3}$ reporting normal neurological examination). Further, some of these individuals seek to be paraplegic. ${ }^{4}$ More rare are reports of individuals who seek a sensory deprivation, such as blindness or deafness. ${ }^{5}$ Nonetheless, all of these cases share the urgency to permanently damage an apparently intact body. ${ }^{6}$

A first description of this condition traces back to a series of letters published in 1972 in the magazine Penthouse. These letters were from erotically-obsessed persons who wanted to become amputees themselves. ${ }^{7}$ However, the first scientific report of this desire only appeared in 1977: Money et al described two cases who had intense desire toward amputation of a healthy limb. ${ }^{7}$ Another milestone was a 2005 study by Michael First, an American psychiatrist, who published the first systematic attempt to describe individuals who desire amputation of a healthy limb. ${ }^{2}$ Thanks to this survey,
Correspondence: Anna Sedda

Department of Brain and Behavioral Sciences, University of Pavia,

Piazza Botta II, 27I00 Pavia, Italy Email anna.sedda@unipv.it 
which included 52 volunteers, a number of key features of the condition are identified: gender prevalence (most individuals are men), side preference (left-sided amputations are most frequently desired), and finally, a preference toward amputation of the leg versus the arm. ${ }^{2}$

So far, the main explanation for this phenomenon has been in favor of a psychological/psychiatric etiology: a pathological desire driven by a sexual compulsion. ${ }^{8}$ However, more recent studies have attempted to identify neural correlates of this condition by means of electrophysiological or neuroimaging techniques. These studies are grounded on the idea that the desire might originate from body representation impairments, rather than from a sexual compulsion. ${ }^{9}$ Further, they have been inspired mainly by First's observation that most of these individuals seek a limb amputation primarily "to feel complete" and not for a sexual reason. ${ }^{2}$

At present, the debate is still open. Understanding whether the desire to amputate a healthy limb is of psychological/psychiatric or neurological origin is a determinant of guiding development of possible treatments, especially considering that most of the approaches that have been tried until now have proven ineffective. ${ }^{2}$

Here, we provide a summary of the evolution of the study of desire for amputation of healthy limbs and for paraplegia. The case reports of Money et al published in 1977, are the starting point, followed by subsequent psychiatric descriptions. Finally, we attempt a first systematization of the available studies that adopt neuroscientific methodologies.

After this summary, and a brief paragraph on the paralysis variant of the desire to modify body representation, we discuss the present limitations of both psychological/psychiatric and neurological approaches, and suggest a new path that could be followed to ensure a full understanding of this peculiar and ethically challenging condition.

\section{Etiological hypotheses Psychiatric/psychological etiology}

A psychological/psychiatric etiology for this condition traces back to the first report of Money et al. ${ }^{7}$ This systematic description illustrates the overwhelming desire to amputate a healthy limb in two individuals. Both were men and physically healthy, with the irrelevant exception of the second individual's right foot, which was smaller than the left, with slightly atrophic calf muscles on the same side. The desire was confined to the left side in the first and to the right side in the second individual. Both reported the onset of the desire around age 11-13 years. The first individual was under stable psychotherapy treatment, referred with sexual concerns about his homosexuality and a deep fear of social disapproval. Further, both individuals affirmed that they did not wish to harm themselves (even though necessary to reach a solution to their desire) and that they were both attracted by asymmetry. The authors diagnosed these individuals as paraphilic, excluding the possibility of paranoid disturbances, and linked the desire to sexual dysfunctions. In their conclusions, the authors suggested that limb amputation could represent a way for the patients to preserve their masculinity while being bisexual. The authors termed the condition apotemnophilia, from the Greek words "apo", which means "away from", and "temno", meaning "piece cut off", and "philia", meaning love, leading to a general meaning of "love for amputation". The authors also suggested that the eroticized willingness to amputate a limb might be a "reversal of an aversive element": "the fear of losing one's limb [...] is metamorphosed into an impulsion to be amputated and to emerge superior or supernormal in achievement". ${ }^{7}$ In later years, Money's approach has been described as a "behavioral point of view on apotemnophilia". ${ }^{8}$ Accordingly, rehearsal of experiences with amputation images during their lifetime is thought to reinforce sexual desire in apotemnophilic individuals. This categorization differs from the psychodynamic approach, which considers the desire for amputation as an erotic manifestation of hatred toward maternal figures. ${ }^{8}$ In any case, this is an a posteriori interpretation, and there are no guarantees that Money himself would agree with this view.

Independent of their categorization within psychodynamic interpretations, these case descriptions also marked the condition as apotemnophilia in subsequent studies. With Money's study, the first label for the desire to be an amputee was born.

Notably, some years later, another report on the same condition suggested an alternative possibility to explain apotemnophilia. ${ }^{10}$ The patient described was a man who wanted to remove one of his legs. The description summarized crucial episodes in the patient's life that were thought to be the origin of the desire: the sight of a boy with a wooden leg during childhood, the attraction toward this boy and the idea that it represented full happiness, the development of homosexual preferences, and finally, a pretending behavior with crutches. The author reported that the crucial motivation of this individual's desire for amputation was to obtain physical and mental well-being. This description focused on the patient's feelings of identity, as he stated that the amputation of the leg was the only way for him to feel complete, and that amputation loses sexual meaning, compared against the relevance of personal identity. Everaerd highlighted that 
his patient did not resemble Money's cases and that there were no problems of sexual identity (such as guilty feelings toward homosexuality and bisexual orientation). ${ }^{10}$

Several other case reports on apotemnophilia were published from 2003-2009 (Table 1). ${ }^{11-16}$ These studies link the desire for amputation to a sexual disturbance, attribute its origin to a paraphilic disturbance, and describe mostly individuals of male gender. Later analysis challenged a genuine apotemnophilic etiology, or highlighted the paucity of clinical information needed to establish a clear diagnosis ${ }^{5}$ in some of these reports. ${ }^{17,18}$ In particular, the scant descriptions of clinical features of these individuals did not allow any firm conclusion to be drawn. Further, some individuals have also been assigned a comorbid psychiatric diagnosis on both Axis I and Axis II (see Bou Khalil and Richa ${ }^{19}$ for details of the diagnoses) (Table 1). However, it should be noted that this psychiatric disturbance is not necessarily the origin of the desire, rather a co-occurrence of another condition, especially in cases of depression and anxiety. A different reasoning applies to schizophrenia and other psychotic disorders that may cause the desire to amputate a limb. In patients affected by these diseases, the desire appears to be externally imposed, usually by God/devil. ${ }^{20}$ Further, even in patients with comorbid schizophrenia, drugs that reduce symptoms associated with this psychiatric condition might not work against the desire for amputation. ${ }^{21}$ These important features allow a distinction to be made between apotemnophilia and schizophrenia.

A robust change of orientation took place in 2005, when Michael First published the first systematic study of a consistent sample of individuals who desired amputation of a healthy limb. ${ }^{2}$ Structured interviews were conducted via telephone with 52 subjects who desired amputation (only four were female). None of the subjects in the study were delusional, and all except one presented the onset of the desire during childhood. As for comorbid psychopathologies, 41 subjects confirmed no psychiatric symptoms, while the others described mild symptoms, mainly anxiety and depression. The same number of subjects, however, reported at least one episode of psychiatric origin during their lives. Fifteen subjects also reported at least one other paraphilic interest, such as cross-dressing or masochism. Importantly, six subjects who obtained amputation were also described, and the author highlighted that this intervention permanently resolved the desire in these individuals.

Notably, First suggested that sexual arousal is a secondary motivation for most individuals; thus, seeking amputation cannot be considered a paraphilia. It was also proposed that this desire not be included within body dysmorphic disorder; individuals seeking amputation are critical of the fact that other persons do not see any defect in their limb appearance, and they acknowledge this, as they do not complain about the physical appearance of the limb. ${ }^{5,22}$ Starting from these assumptions, a parallel is drawn between the desire to amputate a limb and gender identity disorder. ${ }^{2}$ First ${ }^{2,5}$ considers the several similarities between these conditions: feeling uncomfortable with a given anatomical identity, the time of onset being located during childhood, successful treatment being effected by mimicking of the desire, and finally, that paraphilic sexual arousal is obtained through fantasies of the desired identity. Thus, the author concluded that apotemnophilia is not an appropriate label and proposed the term Body Integrity Identity Disorder (BIID), considering that this condition might be an unusual development of self-identity, where the sexual component does not fulfill a primary role. This 2005 survey is considered the first seminal description of a motivation, other than a sexual one, for individuals with an amputation desire, in the absence of a clear psychosis. However, it must be acknowledged that, in 1983, Everaerd had already noticed that sexual compulsion is not necessary to manifest the desire, ${ }^{10}$ even though his work was based on a single individual, rather than on a group study.

Some years later, while reports on apotemnophilia and BIID continued to be published with one or the other nomenclature (Table 1), the 2005 survey was highly criticized by Helen De Preester. ${ }^{8}$ De Preester analyzed in detail the percentages found by First and suggested that looking at these numbers from different perspectives rules out a diverse explanation for the amputation desire. In more detail, De Preester suggested that it is impossible to exclude the sexual component when studying apotemnophilia/BIID. She reanalyzed the data, looking for "pure" cases; in other words, for those individuals in whom the sexual motivation (or the identity motivation) is totally absent. The percentages of "pure apotemnophilic" and "pure identity" individuals appear to be very low (around 10\%). Thus, even though being secondary, the sexual motivation was present in $42 \%$ of the individuals studied by First, if the data are elaborated according to this reasoning. According to De Preester, the fact that the sexual compulsion is secondary does not mean that it is of less importance. This debate on the role of sexual components in apotemnophilia/BIID is still open, and more theoretical than experimental.

More recent work has focused on the possibility of creating a set of criteria to diagnose apotemnophilia and/or BIID. The idea is to establish common ground to facilitate 


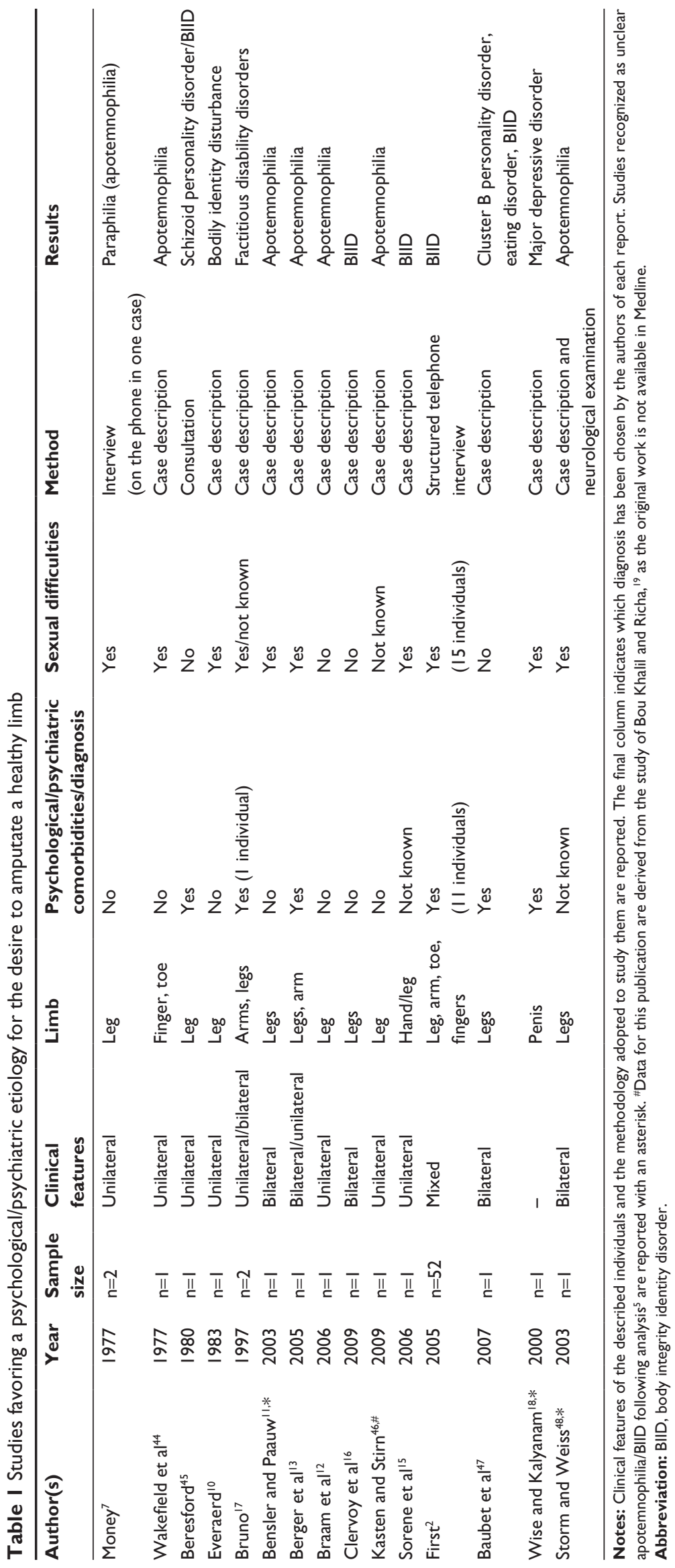


the work of clinicians faced with this condition, as they cannot refer to any manual ${ }^{14}$ and often misdiagnose it, given its rarity and the elusive behavior of patients..$^{19}$ For instance, Ryan and Shaw ${ }^{22}$ highlighted some key features of BIID by comparing it to body dysmorphic disorder. However, these features were not defined as criteria by the authors. In 2012, Bou Khalil and Richa ${ }^{19}$ proposed a set of criteria to establish the presence of BIID, based on a detailed analysis of fourteen case reports available in the literature. It should be noted that this work also included reports identified as "unsure BIID", leaving open the question as to whether the proposed criteria would apply to all individuals, including psychiatric patients. In more detail, these authors identified twelve crucial criteria concerning BIID (or apotemnophilia): age at onset, advanced age at desire, disclosure to family and doctors, predominantly male gender, no predefined sexual orientation, association with gender identity disorder and/or other paraphilia, no psychiatric family history, no predominant laterality for amputation, association with a history of exposition to other amputees during childhood, no association with trauma or sensory impairments of the limb to be amputated, self-amputation behaviors, possible association with personality disorders, and treatment with antidepressant and cognitive behavioral therapies. It can be noticed immediately that while these criteria fit nicely with the case reports reviewed, they might not be suitable for other cases described (see Neurologic etiology section below). First and Fisher ${ }^{5}$ tried to solve this problem by developing criteria that resemble those commonly used in the Diagnostic and Statistical Manual of Mental Disorders (DSM), also including a subtype criterion based on the predominant desired disability. Further, these criteria do not go into detail concerning onset, gender orientation, and encounters with amputated persons in childhood. Rather, the focus is on the desire to be disabled, the distress that follows this desire, and the absence of psychosis. Importantly, criterion D explicitly states: "The desire to become disabled is not primarily motivated by sexual arousal or by any perceived advantages of becoming disabled". ${ }^{5}$ Thus, this new set of criteria also does not fit all case reports.

In summary, psychological/psychiatric explanations for the desire to amputate a healthy limb include two main hypotheses: a sexual compulsion, belonging to the paraphilic core, and an identity disturbance, paralleling gender identity disorder. At present, no new psychological/psychiatric explanations have been proposed; neither has this condition been included in the DSM- 5 classifications.

\section{Neurologic etiology}

Recently, emerging neuroscientific evidence has favored an alternative etiology to the psychiatric/psychological one. Beginning with the work of Ramachandran and McGeoch, ${ }^{9}$ six experimental studies that explored physiological and brain correlates of this condition have highlighted altered cortical architecture (or activity), mainly in the parietal lobe, and atypical behaviors monitored by these same areas (Table 2). These data raise the question as to whether apotemnophilia/BIID could be due to a dysfunction of the anatomical structures devoted to body representation and corporeal awareness.

The first appearance of this neurological hypothesis was in 2007, by Ramachandran and McGeoch. ${ }^{9}$ The authors reasoned that the desire to amputate a healthy limb shares some commonalities with somatoparaphrenia, as individuals with BIID show a left-sided preference for amputation. Somatoparaphrenia is a neuropsychological symptom that emerges mainly after right hemisphere brain damage. ${ }^{23,24} \mathrm{In}$ such cases, patients deny ownership of one limb, usually the left paraplegic arm, maintaining that the arm is not theirs and that their real limb is somewhere nearby. Somatoparaphrenic patients have been shown to remit from their impairments if they are administered cold caloric vestibular stimulation (CVS), due to the effects of this technique on the right parietal lobe. ${ }^{25}$ Consequently, it has been suggested to explore apotemnophilia/BIID by means of functional brain imaging and skin conductance responses. ${ }^{9}$ Ramachandran and McGeoch argued that if symptoms disappear after CVS administration, the condition can be considered to be of neurological origin and, consequently, treatments to abolish the desire should rely on physiological manipulations of specific brain areas.

In 2008, Brang et al presented a preliminary study that explored skin conductance response (SCR) in individuals with the desire to amputate a healthy limb. ${ }^{26}$ The authors adopted a pain paradigm, applying a pinprick above and below the line of desired amputation on each leg of these individuals. Increased SCRs were found for stimuli that contacted the limb selectively below the line of amputation.

Even though this was the first experimental study to focus on more neurological measures, it was still a descriptive report, including only two subjects with quite diverse clinical features: the first desired a right below-knee amputation, while the second desired amputations both below his left knee and below his right thigh.

A second experimental study, with a slightly more consistent group of individuals, followed in 2011. ${ }^{3}$ McGeoch et al 


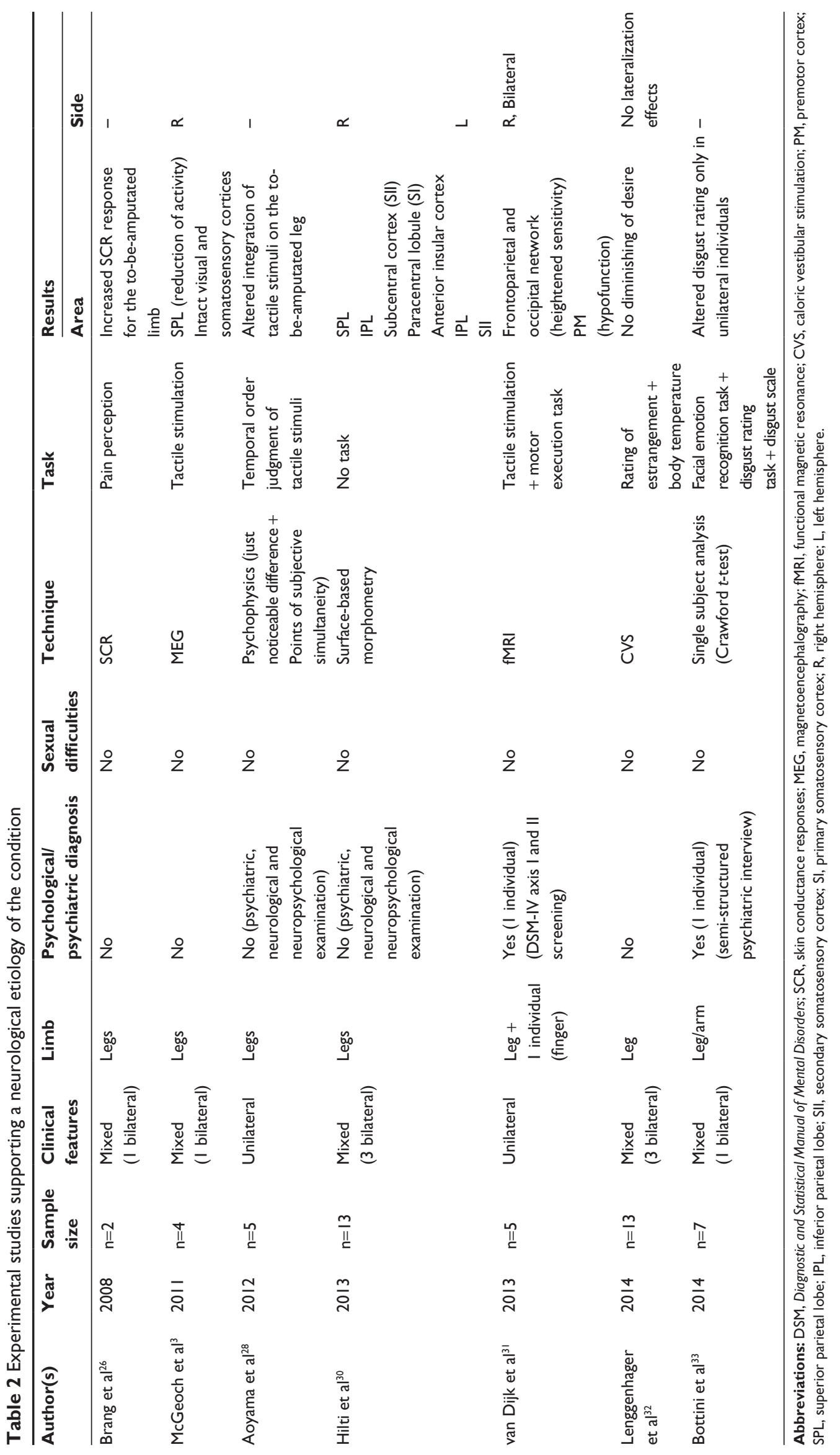


used magnetoencephalography (MEG), in conjunction with a tactile stimulation task, to explore activity of parietal areas. Their findings revealed altered activity in the right parietal lobe. The authors concluded that apotemnophilia/BIID should be termed "xenomelia" and should be included within the right parietal lobe syndromes related to body representation. This new term encompasses the parallel with somatoparaphrenia and means "foreign" (xeno) "limb" (melia) in ancient Greek. It is the third label to be given to the condition in a span of 34 years. It should be noted that, with this label, the idea of a deranged body identity is not abandoned. Rather, it is embodied in a diverse theoretical frame. In this work, there is no mention of a possible sexual component of the condition, even though it should be noted that the authors considered sexual aspects of the desire to amputate a limb in a previous work. ${ }^{27}$

Following the hypothesis of a parietal lobe syndrome, Aoyama et al investigated temporal order judgments in individuals with BIID. ${ }^{28}$ Their experiment was based on the idea that the integrity of body image and of the parietal cortex (in other words, preserved sense of ownership) are necessary in order to correctly evaluate which one of two stimuli consecutively applied to a body part was delivered first. ${ }^{29}$ The findings from analysis of the Points of Subjective Simultaneity (PSS) revealed that individuals with BIID perceive stimuli that are more distal as occurring first. In other words, stimuli that are located within the to-be-amputated leg are perceived first. Contrastingly, PSS should be in favor of proximal body parts, due to neural transmission times being faster than from distal areas. The authors argued that this evidence confirms that the desire to amputate a healthy limb is a parietal lobe syndrome, as their subjects had no sensory impairments that could explain the results.

In partial agreement, Hilti et al in 2013, reported structural differences between control subjects and individuals who desired to amputate a healthy limb, and highlighted differences in the right superior parietal lobe (SPL) - also in the right primary and secondary somatosensory cortices, and in the anterior insula. ${ }^{30}$ This study included no active tasks, as the aim was only to explore cortical architecture.

A functional magnetic resonance imaging (fMRI) experiment was conducted in the same year, to compare brain activity between limbs which felt "owned" and limbs felt "to-be-amputated" during a tactile stimulation and a motor execution task. ${ }^{31}$ Firstly, results of the tactile stimulation task, analyzed in general terms (activity related to both legs of individuals with BIID versus controls), highlighted a different responsivity in the somatosensory network, largely encompassing the frontoparietal network (the dorsal premotor cortex [PMd]; the precentral and postcentral gyri, including the somatosensory cortex; the SPL, bilaterally; the right ventral premotor cortex $[\mathrm{PMv}]$; the insular cortex; and the supramarginal gyrus) and the occipitotemporal cortex (the lateral occipital cortex; the precuneus; the inferior temporal cortex; the fusiform gyrus; and the cerebellum, bilaterally). Importantly, no brain regions showed significantly reduced responsivity. Secondly, the authors took into account neural activity as a function of the leg (the "to-be-amputated" versus the "owned" leg, in comparison to the corresponding leg in control subjects). These contrasts revealed reduced activation in the contralateral PMv and PMd, compared to controls. No significant increase in brain activity was shown in any area. These results suggest that the feeling of disownership in this condition is associated with altered somatosensory processing. On the other hand, results from the motor execution task did not show significant differences, neither considering a general comparison between controls and individuals with apotemnophilia/BIID/xenomelia, nor taking into account leg ownership.

Finally, a recent study disconfirmed the hypothesis of Ramachandran and McGeoch: symptoms related to the desire for amputation did not disappear after CVS: the converse of what happens with somatoparaphrenic patients. ${ }^{32}$ These results seem to preclude this condition being as simple as a "developmental somatoparahrenia" and cast new doubt on a pure neurologic etiology, despite the promising results of the other imaging studies.

Finally, a recent study ${ }^{33}$ explored the neurological hypothesis, starting from a different perspective. In this work, the authors administered a set of tests to 7 individuals with BIID, which were aimed at exploring facial expression: recognition and disgust responses. The basic premise was that the insula, indicated in previous studies as a possibly dysfunctional area, is involved in body representation ${ }^{34}$ and also in emotional processing. ${ }^{35}$ A second starting point, which convinced the authors to follow this path, is that psychiatric disturbances are usually comorbid with emotion recognition impairments. ${ }^{36}$ Differently from previous studies that focused on body representation per se and neglected the sexual and psychiatric components, this study adopted a neuropsychological methodology and explored both the neurological and psychiatric etiologies. It is relevant that the authors treated the data as single cases, avoiding the problem of grouping together individuals with different clinical features. The results of this study showed absence of emotion recognition and also expression impairments, in all individuals. However, 
subjects who sought unilateral amputation showed a different pattern in regard to the evaluation of images depicting amputated limbs (not amputated individuals, but amputated limbs, excluding any sexual component of attraction toward an amputee). These individuals rated these images as "not disgusting" - a behavior converse to what can be expected from known models of disgust toward body envelope violations. ${ }^{1,37}$ Such models postulate that disgust is a defensive reaction toward danger; consequently, violations of the body representation elicit this emotion. ${ }^{1,37}$ It appears that BIID is characterized by a compromising of emotion, selectively related to the body, but only in some individuals who share specific clinical features.

\section{Paralysis version}

The studies we reviewed present individuals willing to amputate a limb, independent of the underlying etiology. However, two recent surveys highlight that the desire to modify the body might also present with non-amputation variants. ${ }^{4,21}$ Before these two studies, in 1997, Bruno ${ }^{17}$ noticed that individuals with apotemnophilia could present a peculiar desire to be paralyzed. He described in detail the case of a woman who was willing to be paralyzed and who pretended to be so, using a wheelchair. However, Bruno did not include this desire within apotemnophilia. Rather, he proposed a psychological concept to explain not only a fascination for being with disabled persons, but also a desire to pretend to be disabled, and even to become disabled: "factitious disability disorders"17 (see First and Fisher ${ }^{5}$ for a discussion).

Alternatively, two more recent reports suggest that the desire to be paralyzed is a variant of apotemnophilia/BIID. ${ }^{4,21}$ It should be noted that, in these cases, the adoption of the term xenomelia is not appropriate, as individuals who want to become paraplegic do not want to lose their limbs., ${ }^{4,21}$

These surveys adopted questionnaires developed ad hoc to screen individuals about their desire, age at its onset, sexual compulsions, comorbid psychiatric pathologies, and other relevant clinical features.

Forty-four percent of the 54 individuals recruited by Blom et $\mathrm{al}^{21}$ reported a wish to be disabled, but not through amputation. Twenty-four of these individuals (seven females; $29 \%$ of the sample) desired to become paralyzed (about $44 \%$ of the entire sample) and indicated the exact location of the desired paralysis. The majority of individuals who desired paralysis were heterosexual, while seven individuals were homosexual and four were bisexual. Further, 11 individuals also presented with sexual arousal that was related to seeing persons with the desired disability. The same number of interviewed persons described a psychiatric comorbidity during their lifetime (eg, depression, anxiety, psychosis). The authors suggested that those individuals with the paralysis version of the desire did not differ in clinical features from those wanting an amputation. They concluded that this variant is a manifestation of the same desire. However, the authors did not provide a hypothesis to explain the existence of two variants of the same desire.

In their study, Giumarra et $\mathrm{al}^{4}$ enrolled 16 individuals with the paralysis variant and reached a conclusion in favor of a variability that can be attributed to gender differences. As for the clinical features (psychiatric comorbidities, sexual arousal), the rates are similar between the two studies, equally supporting the inadequacy of psychotic or mood disorders to explain the desire. Giumarra et al also reported that all participants who wanted a paralysis were able also to define a clear demarcation line for the desired spinal cord injury, as do subjects seeking amputation. Secondly, a higher proportion of women $(37.5 \%)$ was found to have this desire variant. Thus, the authors hypothesized that the desire to be paralyzed is neurologically-based, as it is for amputation, and suggested that women are more inclined toward paralysis, as they have a less marked lateralization of the corpus callosum, which could lead to a "bilateral desire".

While this explanation seems appealing, it should be noted that the paralysis variant also manifests in men (though to a lesser degree than in woman, ${ }^{21}[15 \%$ versus $29 \%$, respectively]). Thus, a different lateralization of the body representation, as a function of gender, does not fully account for all of the cases.

\section{Conclusion and future directions}

In this review, we aimed to systematize the available studies of individuals who manifest a willingness to remove one healthy limb, to become paraplegic, or to obtain other motor or sensory disabilities. To understand the complexity of this condition, and witnessing the continuing debate around it, the reader can appreciate the changes in terminology that have been adopted during the 37 years that have passed since the first report. This semantic phenomenon mirrors a shift in the methodology adopted and, accordingly, the diverse disciplines to have approached this condition. From the starting label "apotemnophilia", authors have turned to "BIID" and, very recently, have moved to "xenomelia". While the first term accents psychiatric/psychological features, the last label emphasizes a more neuroscientific taxonomy. In between them, BIID parallels gender identity disorder, at the same time leaving open the possibility of a neurological correlate. 
This path in the nomenclature highlights the absence of agreement concerning this condition, as well as the lack of a composite and uniform approach toward exploring it.

On one side, psychological/psychiatric explanations can be classified into two main categories. A first line of approach considers the desire for amputation as belonging to paraphilic disturbances, and identifies sexual arousal and compulsion as the first motivation. The second point of view includes this desire within a new disturbance, which parallels gender identity disorder but is related to a dysfunctional representation of the body, not necessarily caused by an impaired neural correlate. Thus, the psychological/psychiatric approach provides quite divergent results, even while using the same methods (interviews and descriptions of individuals) and considering the same clinical features (age at onset, manifestation of the desire, sexuality, etc).

The most recent hypothesis on the origin of this condition is neurologic. At present, only six studies have tested this idea experimentally, using different samples and diverse techniques (Table 2). There is no overlap between techniques across these studies; they range from imaging methods (fMRI, ${ }^{31} \mathrm{MEG},{ }^{3}$ and surface-based morphometry ${ }^{30}$ ) to psychophysics, to physiological measures/manipulations $\left(\mathrm{SCR},{ }^{26} \mathrm{CVS}^{32}\right)$. This is less true of the samples included. On one side, unilateral and bilateral individuals are usually included in the same group, with just two studies ${ }^{28,31}$ having enrolled only unilateral subjects. On the other side, none of these studies have included individuals with a desire for amputation of upper limbs, nor with a desire for paraplegia. These clinical features, together with the desire to be blind or deaf, ${ }^{5}$ do not necessarily imply similar results in all cases. For instance, the paradigms adopted for a desire that is focused on the leg do not appear to be suitable for exploring the desire for blindness, as there would be no theoretical reasons to hypothesize a tactile dysfunction (neither sensory, nor in terms of altered temporal judgments) or any difference concerning sensorimotor networks and the premotor cortex.

Ideally, the adoption of different techniques and methods should provide strength, if the findings are to point convincingly toward the same pattern (ie, a parietal lobe dysfunction or syndrome). However, the few available experiments show discordant results, as do also the psychological/psychiatric descriptions and surveys. Notably, neuroscientific methods are perceived as more objective than those with a psychological/psychiatric frame. But, in this case, they lead to the same confounding results. A striking example concerns results on primary and secondary somatosensory cortices. According to $\mathrm{McGeoch}$ et $\mathrm{al}^{3}$ these areas are spared in xenomelia, while Hilti et $\mathrm{al}^{30}$ suggest more caution, and highlight structural differences. Possibly, the employment of diverse techniques (in one case, MEG plus one active task; ${ }^{3}$ in another case, surface-based morphometry ${ }^{30}$ ) can explain this relevant discrepancy. However, even though a difference in structural anatomy does not necessarily mean a hypofunctioning, the study that adopted fMRI and a tactile stimulation task also demonstrated a dysfunction of the somatosensory network. Despite the discrepancies between neurologicoriented studies, some commonalities also emerged: notably, the MEG paper found reduced right SPL activity, which was also one of the cortical areas found to be unduly thin in the surface-based morphometry paper. Possibly, future studies should concentrate on further exploring these areas, which consistently emerge from studies, even when they adopt different methodologies.

In summary, study results do not completely converge and cannot be considered conclusive evidence of a neurologic etiology for BIID. Neither are the results of the psychological/psychiatric investigations more convincing in favor of a sexual motivation or an identity dysfunction.

At present, it can only be concluded that some neurostructural or/and neurofunctional differences exist between individuals who seek to amputate a healthy limb and those who do not. Further, individuals with this condition show psychiatric comorbidities during their lifetime, even though not sufficient to explain their desire. Finally, some clinical features, such as the age at onset, the presence/absence of sexual arousal for amputation and amputees, pretending behaviors, and the lateralization of the desire appear to be crucial to understanding this condition. Nevertheless, it should be kept in mind that it still not known whether all of these features also apply to blindness/deafness and paraplegia variants, especially as far as brain differences are concerned. Even though the current proposal, based on clinical features and interviews, is to assume that all variants are part of the same syndrome, it appears clear that there is no available evidence that these groups share a common pathogenesis. In particular, no neuroimaging studies have yet been conducted. Thus, it seems premature to consider this hypothesis as final.

Second, are these brain differences a cause or a consequence of BIID? Considering (for instance) CVS, it is known that this manipulation is effective on somatoparaphrenia. ${ }^{25,23}$ But, the key point is that (usually) this neuropsychological impairment results from brain damage in adult individuals. As for the desire to amputate a healthy limb or to be paraplegic, it has not yet been proven whether brain differences are 
preexisting or follow from this atypical behavior. ${ }^{5}$ It is the classical dilemma of nature or nurture: which comes first? If the observed brain correlates are the consequence of a repetitive behavior (as indicated by the psychiatric etiology), ${ }^{5,8}$ then it is no surprise that such a technique is ineffective. Altering the vestibular components of sensory perception and body representation ${ }^{25}$ is not enough, if a condition originates from complex psychological components. ${ }^{6}$ Further, there is no theoretical reason to expect a vestibular modulation from a sexual compulsion. Nevertheless, the ineffectiveness of CVS does not prove the converse: that the desire to modify one's own body representation is not a parietal lobe syndrome. One possibility is that, in individuals with the desire to amputate one healthy limb, the changes are too long-standing and entrenched. Thus, the evidence that they do not respond to treatment with CVS does not mean that the neurological hypothesis is wrong. Conversely, in somatoparaphrenia, the sufferers often tend to have a condition that is short-lived, reverses spontaneously, and is more amenable to CVS treatment.

Possibly, one way to solve this puzzle would be also to conduct genetic studies, which might help determine whether there is a pattern of genes that influences the development of an altered body representation in individuals with this condition. ${ }^{21}$ The "nature or nurture" question needs to be answered at some point, and one can anticipate that the path to finding the answer will be long and difficult.

Clearly, the desire to be disabled poses social, ethical, and clinical problems that are strongly debated by scientists and philosophers, as the freedom to act on one's own body is restricted by law. ${ }^{38-42}$ The request for amputation or paraplegia or other disabilities is not a soft one. Serious legal issues could arise if individuals are allowed to fulfill these requests before any clear etiology and clinical classification has been established. Second, the fact that these requests are so diverse complicates the story, suggesting that a mere sense of disownership cannot explain all the conditions. Systematic postamputation studies are still not available; thus, it is unknown whether relief at finally feeling "complete" really overcomes the inevitable collateral effects of amputation. Importantly, there are also cases in which amputation does not resolve the desire $\left(\mathrm{see}^{19}\right)$.

Regardless of all the theoretical disagreement, this condition starts very early in life, no matter which etiology proves true, and it tends to exacerbate over time, causing sufferers terrible distress and driving them toward definitive and irreparable interventions. Thus, further efforts to understand this new condition will undeniably be of value, not only at a theoretical level but especially also considering the ethics beyond it. A strict separation between the methods and theories of psychology/psychiatry and neuroscience does not appear to be the solution to understanding such a complex condition. Conversely, integrating neuroscientific and psychological/psychiatric methods appears to be feasible and desirable, even though challenging. Neuroimaging studies that include both theoretical premises should be preferred, comprising experiments that explore both body representation and psychological/sexual components. Further, the samples to be studied should include diverse variants of the desire, to elucidate whether they show differences.

In conclusion, the 37 years of study of this desire to be disabled have brought to light a still secretive condition (but possibly not-so-rare, considering the increasing numbers of individuals included in the studies), which appears challenging to understand, even using state-of-the-art technologies. Much more effort is needed to find a solution and, finally, a treatment for the distress these individuals experience. This still-obscure condition needs a multidisciplinary approach to go beyond the "simple" clinical/experimental frame, and requires a much more complex model that also includes social and ethical aspects. ${ }^{42}$

\section{Disclosure}

None of the authors have any conflicts of interest to disclose.

\section{References}

1. Rozin P, Haidt J, Fincher K. Psychology. From oral to moral. Science. 2009;323(5918):1179-1180.

2. First MB. Desire for amputation of a limb: paraphilia, psychosis, or a new type of identity disorder. Psychol Med. 2005;35(6):919-928.

3. McGeoch PD, Brang D, Song T, Lee RR, Huang M, Ramachandran VS. Xenomelia: a new right parietal lobe syndrome. J Neurol Neurosurg Psychiatry. 2011;82(12):1314-1319.

4. Giummarra MJ, Bradshaw JL, Hilti LM, Nicholls ME, Brugger P. Paralyzed by desire: a new type of body integrity identity disorder. Cogn Behav Neurol. 2012;25(1):34-41.

5. First MB, Fisher CE. Body integrity identity disorder: the persistent desire to acquire a physical disability. Psychopathology. 2012;45(1): 3-14.

6. Sedda A. Body integrity identity disorder: from a psychological to a neurological syndrome. Neuropsychol Rev. 2011;21(4):334-336.

7. Money J, Jobaris R, Furth G. Apotemnophilia: two cases of self-demand amputation as a paraphilia. J Sex Res. 1977;13(2):115-125.

8. De Preester H. Merleau-Ponty's sexual schema and the sexual component of body integrity identity disorder. Med Health Care Philos. 2013;16(2):171-184.

9. Ramachandran VS, McGeoch P. Can vestibular caloric stimulation be used to treat apotemnophilia? Med Hypotheses. 2007;69(2):250-252.

10. Everaerd W. A case of apotemnophilia: a handicap as sexual preference. Am J Psychother. 1983;37(2):285-293.

11. Bensler JM, Paauw DS. Apotemnophilia masquerading as medical morbidity. South Med J. 2003;96(7):674-676.

12. Braam AW, Visser S, Cath DC, Hoogendijk WJ. Investigation of the syndrome of apotemnophilia and course of a cognitive-behavioural therapy. Psychopathology. 2006;39(1):32-37. 
13. Berger BD, Lehrmann JA, Larson G, Alverno L, Tsao CI. Nonpsychotic, nonparaphilic self-amputation and the internet. Compr Psychiatry. 2005; 46(5):380-383.

14. Kasten E. [Body Integrity Identity Disorder (BIID): interrogation of patients and theories for explanation]. Fortschr Neurol Psychiatr. 2009; 77(1):16-24. German.

15. Sorene ED, Heras-Palou C, Burke FD. Self-amputation of a healthy hand: a case of body integrity identity disorder. J Hand Surg Br. 2006; 31(6):593-595.

16. Clervoy P, Vautier V, Naudin J. [Body identity integrity disorder: clinical questioning and ethical issues]. Ann Med Psychol (Paris). 2009; 167(4):251-255. French.

17. Bruno RL. Devotees, pretenders, and wannabes: two cases of factitious disability disorder. Sex Disabil. 1997;15(4):243-260.

18. Wise TN, Kalyanam RC. Amputee fetishism and genital mutilation case report and literature review. J Sex Marital Ther. 2000;26(4): 339-344.

19. Bou Khalil R, Richa S. Apotemnophilia or body integrity identity disorder: a case report review. Int J Low Extrem Wounds. 2012;11(4): 313-319.

20. Schlozman SC. Upper-extremity self-amputation and replantation: 2 case reports and a review of the literature. J Clin Psychiatry. 1998; 59(12):681-686

21. Blom RM, Hennekam RC, Denys D. Body integrity identity disorder. PLoS One. 2012;7(4):e34702.

22. Ryan CJ, Shaw T, Harris AW. Body integrity identity disorder: response to Patrone. J Med Ethics. 2010;36(3):189-190.

23. Bottini G, Sedda A, Ferrè ER, Invernizzi P, Gandola M, Paulesu E. Productive symptoms in right brain damage. Curr Opin Neurol. 2009; 22(6):589-593.

24. Gandola M, Invernizzi P, Sedda A, et al. An anatomical account of somatoparaphrenia. Cortex. 2012;48(9):1165-1178.

25. Bottini G, Gandola M, Sedda A, Ferrè ER. Caloric vestibular stimulation: interaction between somatosensory system and vestibular apparatus. Front Integr Neurosci. 2013;7:66.

26. Brang D, McGeoch PD, Ramachandran VS. Apotemnophilia: a neurological disorder. Neuroreport. 2008;19(13):1305-1306.

27. Ramachandran VS, Brang D, McGeoch PD, Rosar W. Sexual and food preference in apotemnophilia and anorexia: interactions between 'beliefs' and 'needs' regulated by two-way connections between body image and limbic structures. Perception. 2009;38(5): 775-777.

28. Aoyama A, Krummenacher P, Palla A, Hilti LM, Brugger P. Impaired spatial-temporal integration of touch in xenomelia (body integrity identity disorder). Spat Cogn Comput. 2012;12(2-3):96-110.

29. Moseley GL, Olthof N, Venema A, et al. Psychologically induced cooling of a specific body part caused by the illusory ownership of an artificial counterpart. Proc Natl Acad Sci U S A. 2008;105(35):13169-13173.
30. Hilti LM, Hanggi J, Vitacco DA, et al. The desire for healthy limb amputation: structural brain correlates and clinical features of xenomelia. Brain. 2013;136(Pt 1):318-329.

31. van Dijk MT, van Wingen GA, van Lammeren A, et al. Neural basis of limb ownership in individuals with body integrity identity disorder. PLoS One. 2013;8(8):e72212.

32. Lenggenhager B, Hilti L, Palla A, Macauda G, Brugger P. Vestibular stimulation does not diminish the desire for amputation. Cortex. 2014;54: 210-212.

33. Bottini G, Brugger P, Sedda A. Is the desire for amputation related to disturbed emotion processing? A multiple case study analysis in BIID. Neurocase. 2014. Epub 2014 Mar 28.

34. Berlucchi G, Aglioti SM. The body in the brain revisited. Exp Brain Res. 2010;200(1):25-35.

35. Jabbi M, Bastiaansen J, Keysers C. A common anterior insula representation of disgust observation, experience and imagination shows divergent functional connectivity pathways. PLoS One. 2008;3(8):e2939.

36. Kohler CG, Hoffman LJ, Eastman LB, Healey K, Moberg PJ. Facial emotion perception in depression and bipolar disorder: a quantitative review. Psychiatry Res. 2011;188(3):303-309.

37. Rozin P, Fallon AE. A perspective on disgust. Psychol Rev. 1987;94(1) 23-41

38. Dua A. Apotemnophilia: ethical considerations of amputating a healthy limb. J Med Ethics. 2010;36(2):75-78.

39. Jotkowitz A, Zivotofsky A. Body integrity identity disorder (BIID) and the limits of autonomy. Am J Bioeth. 2009;9(1):55-56.

40. Bridy A. Response to Muller. Body integrity identity disorder (BIID). Am J Bioeth. 2009;9(9):W8.

41. Muller S. Body integrity identity disorder (BIID) - is the amputation of healthy limbs ethically justified? Am J Bioeth. 2009;9(1):36-43.

42. Patrone D. Disfigured anatomies and imperfect analogies: body integrity identity disorder and the supposed right to self-demanded amputation of healthy body parts. J Med Ethics. 2009;35(9):541-545.

43. Brugger P, Lenggenhager B, Giummarra MJ. Xenomelia: a social neuroscience view of altered bodily self-consciousness. Front Psychol. 2013;4:204.

44. Wakefield PL, Frank A, Meyers RW. The hobbyist: a euphemism for selfmutilation and fetishism. Bull Menninger Clin. 1977;41:539-552.

45. Beresford TP. The dynamics of aggression in an amputee. Gen Hosp Psychiatry. 1980;9:219-225.

46. Kasten E, Stirn A. Body Integrity Identity Disorder (BIID). Z Psychiatr Psychol Psychother. 2009;57:55-61. German.

47. Baubet T, Gal B, Doncker-Viry D, Masquelet AC, Gatt MT, Moro MR [Apotemnophilia as a contemporary frame for psychological suffering] Encephale. 2007;33:609-615. French.

48. Storm D, Weiss MD. Self-inflicted tourniquer paralysis mimicking acute demyelinating polyneuropathy. Muscle Nerve. 2003;27:631-635.
Neuropsychiatric Disease and Treatment

\section{Publish your work in this journal}

Neuropsychiatric Disease and Treatment is an international, peerreviewed journal of clinical therapeutics and pharmacology focusing on concise rapid reporting of clinical or pre-clinical studies on a range of neuropsychiatric and neurological disorders. This journa is indexed on PubMed Central, the 'PsycINFO' database and CAS,

\section{Dovepress}

and is the official journal of The International Neuropsychiatric Association (INA). The manuscript management system is completely online and includes a very quick and fair peer-review system, which is all easy to use. Visit http://www.dovepress.com/testimonials.php to read real quotes from published authors. 